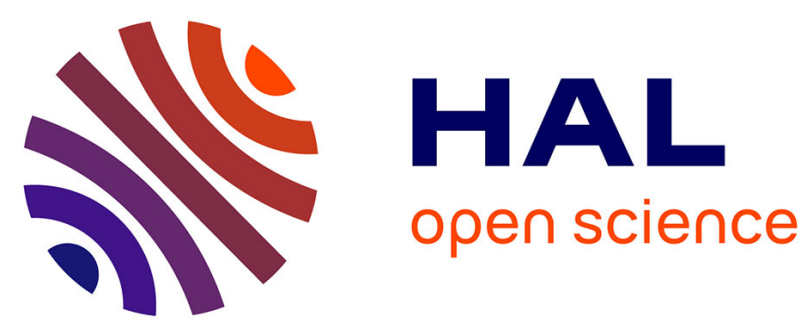

\title{
Caractérisation du potentiel toxique des eaux urbaines par bioessais - Cas de l'agglomération parisienne
}

R. Moilleron, C. Morin, L. Paulic, A. Marconi, V. Rocher, R. Mailler, Adèle Bressy, L. Garrigue-Antar

\section{- To cite this version:}

R. Moilleron, C. Morin, L. Paulic, A. Marconi, V. Rocher, et al.. Caractérisation du potentiel toxique des eaux urbaines par bioessais - Cas de l'agglomération parisienne. Techniques Sciences Méthodes , 2020, 12, pp.175-194. 10.36904/tsm/201912175 . hal-02935113

\section{HAL Id: hal-02935113 \\ https://hal.science/hal-02935113}

Submitted on 10 Sep 2020

HAL is a multi-disciplinary open access archive for the deposit and dissemination of scientific research documents, whether they are published or not. The documents may come from teaching and research institutions in France or abroad, or from public or private research centers.
L'archive ouverte pluridisciplinaire HAL, est destinée au dépôt et à la diffusion de documents scientifiques de niveau recherche, publiés ou non, émanant des établissements d'enseignement et de recherche français ou étrangers, des laboratoires publics ou privés. 
1 CARACTERISATION DU POTENTIEL TOXIQUE DES EAUX URBAINES PAR BIOESSAIS - CAS DE

2 L'AGGLOMERATION PARISIENNE

3 CHARACTERIZATION OF THE TOXIC POTENTIAL OF URBAN WATER BY BIOASSAYS - CASE OF

4 THE PARIS CONURBATION

5 Moilleron Régis ${ }^{1^{\star}}$, Morin Christophe ${ }^{1}$, Paulic Laurent ${ }^{2}$, Marconi Anthony ${ }^{2}$, Rocher Vincent ${ }^{3}$, Mailler

6 Romain ${ }^{3}$, Bressy Adèle ${ }^{4}$, Garrigue-Antar Laure, 1

${ }^{1}$ Leesu, 61 avenue du Général de Gaulle, 94010 Créteil Cedex

2 Tame-Water (ex TRONICO VigiCell), 3 Rue Jean Jaurès, 85000 La Roche-sur-Yon ${ }^{3}$ SIAAP - Direction Innovation Environnement, 92700 Colombes

11 *moilleron@u-pec.fr, 0182392124

12 CATEGORIE DE L'ARTICLE : Retour d'expérience

13 FORMAT DE L'ARTICLE : Article scientifique 


\section{Résumé}

Dans un cadre réglementaire, la qualité d'une eau est souvent évaluée en comparant des concentrations de substances individuelles à des seuils comme, par exemple, les normes de qualité environnementale. Cette approche, bien que robuste, présente certaines limites. La seule information sur la présence de contaminants ne suffit pas à quantifier l'impact ou le potentiel toxique de ces eaux; l'information reste " individuelle » à l'échelle de substances, pour lesquelles suffisamment de données écotoxicologiques existent, sans permettre d'évaluer l'effet cocktail qui pourrait en résulter. Les bioessais sont des méthodes globales et intégrées qui fournissent des informations sur le potentiel toxique de l'échantillon considéré voire sur la toxicité spécifique de certains groupes de substances. Notre démarche a donc consisté à suivre le potentiel toxique de différents échantillons d'eaux urbaines (effluent hospitalier, eaux usées à l'exutoire de deux sous-bassins de la ville de Paris, eaux usées en entrée de station d'épuration et eau épurée, déversoirs d'orage) en utilisant trois panels: Toxicité générale ( 8 bioessais sur algues, bactéries, champignons et cellules humaines), Génotoxicité ( 3 bioessais sur bactéries et cellules humaines), Perturbation endocrinienne (6 bioessais sur cellules humaines). Les résultats montrent que les déversoirs d'orage apportent un excès de toxicité au milieu récepteur. Sur l'ensemble des émissaires, toutes les dimensions de la toxicité ont, à un moment ou un autre, été observées. La comparaison entre entrée et sortie de station d'épuration semblerait indiquer que l'abattement des paramètres physico-chimiques ne se retrouve pas pour les indicateurs de la toxicité. Cependant, des études complémentaires sur ce type d'échantillons sont nécessaires pour confirmer ou non cette première tendance.

Mots-clés : Bioessais, eaux usées urbaines, génotoxicité, toxicité, perturbateurs endocriniens

\section{Abstract}

In a regulatory framework, water quality is often assessed by comparing concentrations of individual substances with thresholds such as environmental quality standards. This approach, although robust, presents some limitations. Information on the presence of contaminants alone is not sufficient to quantify the impact or toxic potential of these waters; the information remains "individual" at the level of substances for which sufficient ecotoxicological data exist, without allowing the potential cocktail effect to be assessed. Bioassays are global and integrated methods that provide information on the toxic potential of the sample under consideration and even on the specific toxicity of certain groups of substances. Our approach therefore consisted in monitoring the toxic potential of various urban water samples (hospital effluent, wastewater at the outlet of two sub-basins of the City of Paris, wastewater at the inlet of a wastewater treatment plant and treated water, combined sewer overflows) using three panels: General toxicity (8 bioassays on algae, bacteria, fungi and human cells), Genotoxicity (3 bioassays on bacteria and human cells), Endocrine disruption (6 bioassays on human cells). The results show that combined sewer overflows bring excess toxicity to the receiving environment. Indeed, all the dimensions of toxicity have, at one time or another, been observed in the emissaries as a whole. The comparison between the inlet and outlet of wastewater treatment plants seems to indicate that the reduction found for the physico-chemical parameters is not observed for toxicity indicators. However, further studies on these types of samples are required to confirm, or not, this trend.

53 Keywords: Bioassays, urban wastewater, genotoxicity, toxicity, endocrine disruptors 
Introduction

56 Depuis l'implémentation de la Directive cadre sur l'eau ou DCE (European Commission, 2000) pour prévenir et réduire la pollution des eaux de surface, les concentrations dans le milieu sont comparées aux Normes de Qualité Environnementale (NQE). Les NQE sont utilisées pour évaluer (i) l'état chimique, qui concerne les substances "prioritaires " et "dangereuses prioritaires" de la DCE et (ii) l'état chimique dans l'état écologique, qui concerne les polluants spécifiques de l'état écologique (PSEE) de la DCE. Dans ce contexte, au niveau européen, 53 substances définissent le bon état chimique et environ une trentaine de substances contribuent à l'état écologique des eaux de surface. Des informations pertinentes sont nécessaires pour évaluer la qualité des eaux de surface : niveaux de contamination par les substances prioritaires mais aussi potentiel toxique que cette contamination génère.

Les approches par mesures physico-chimiques traditionnelles, bien que robustes, montrent leurs limites car trop souvent cantonnées aux espèces imposées par la réglementation et ne considérant pas les métabolites. De plus, la seule information sur la présence de contaminants ne suffit pas à quantifier l'impact ou le potentiel toxique de ces eaux; elle reste "individuelle " à l'échelle de chaque substance sans permettre d'évaluer l'effet cocktail qui pourrait en résulter. Aussi, il est nécessaire d'évaluer la toxicité ou le potentiel toxique via des tests adaptés intégratifs, devant être suffisamment sensibles pour "répondre " à des concentrations proches des NQE, voire en dessous de ce seuil, et fournir des éléments en termes de potentiel toxique « global » (Wernersson et al., 2015). En particulier, les bioessais représentent une avancée dans la surveillance de la qualité de l'eau dans l'environnement (Jarošová et al., 2014 ; Brack et al., 2015 ; Brack et al., 2019). Les méthodes basées sur le suivi des effets biologiques (bioessais in vitro, in vivo et biomarqueurs) sont des approches pertinentes, efficaces et complémentaires des analyses physicochimiques (Leusch et al., 2014 ; Nelson et al. 2007 ; Tang et al. 2014 ; Prasse et al., 2015 ; Wernersson et al. 2015).

Les bioessais ont été régulièrement utilisés afin d'évaluer la toxicité des rejets de station de traitement des eaux usées (STEU). Loos et al. (2013) ont analysé dans les effluents de 75 STEU européennes 156 substances polaires organiques et évalué l'activité œstrogénique ainsi que la toxicité aiguë mettant en évidence une toxicité significative de certains effluents. Cargouët et al. (2004) ont de même suivi le potentiel œstrogénique des eaux usées brutes, des eaux traitées en sortie de station de traitement des eaux usées (STEU) et dans le milieu récepteur. Aït-Aïssa et al. (2017) rapportent que les sources principales de potentiel de perturbation endocrinienne sont les eaux usées et que celui-ci est fortement éliminé par les procédés de traitements. Cependant, il persiste un transfert des composés œstrogéniques vers le milieu naturel par les STEU qui pourrait être réduit par la mise en place de traitements tertiaires. Du Pasquier et al. (2015) ont par exemple montré l'existence de variabilité temporelle du potentiel de perturbation endocrinienne en entrée et en sortie d'une unité de traitement tertiaire par charbon actif.

Toutefois, les études utilisant les bioessais ne permettent généralement pas d'identifier l'origine de la toxicité mais conduisent à la caractérisation de celle-ci. Pour comprendre l'origine de la toxicité observée, une étude plus en amont dans le cycle de l'eau, au niveau des rejets urbains, par temps sec (rejets de station d'épuration) comme par temps de pluie (déversoirs d'orages traités ou non, rejets de station d'épuration en configuration " pluie », etc.) permettrait d'identifier l'origine des apports toxiques au milieu récepteur et 
du cycle en s'attachant à suivre l'évolution du potentiel toxique des eaux urbaines résiduaires de leur génération au niveau des particuliers comme à celui des industriels raccordés, à leur rejet vers le milieu récepteur après traitement. Cette approche est complémentaire de celle proposée dans le cadre du diagnostic amont préconisé dans la note technique du 12 août $2016^{1}$ qui se concentre sur la recherche de 96 substances (Lagarrigue et al., 2018 ; Moilleron et al. 2019). La démarche retenue dans le cadre de cette étude, dite "par matrice», se rapproche de celle de l'Évaluation des Risques Écotoxicologiques ou approche ÉRÉ (Boillot, 2008) qui consiste à évaluer l'impact sur les écosystèmes d'un mélange. Cette démarche s'appliquant au milieu urbain et non au milieu récepteur est originale surtout à l'échelle de l'agglomération parisienne. Nous nous sommes appuyés sur la plateforme de services en bioessais cellulaires in vitro de Tame-Water, partenaire de cette action, qui étudie une diversité d'effets (létaux, sublétaux, marqueurs spécifiques, etc.) sur plusieurs organismes (algues, bactéries, champignons et cellules humaines). En effet, relever un seul type d'effet sur un seul organisme peut conduire à nombre de faux négatifs, dans la mesure où la toxicité mesurée peut ne pas s'exprimer dans les conditions de réalisation (temps d'exposition, paramètre mesuré) d'un bioessai donné (Charissou et al., 2006 ; Connon et al., 2012). II en est de même pour l'organisme modèle qui peut s'avérer pas ou peu sensible à une toxicité donnée. In fine notre objectif est de présenter les limites et performances des bioessais pour évaluer l'impact des polluants émergents sur le vivant par leur application systématique à différents types d'eau rencontrés en milieu urbain.

\section{Matériels et méthodes}

115 Les bioessais sélectionnés permettent de mettre en évidence la présence de polluants de différentes 116 origines pouvant induire des effets les plus variés possibles sur des organismes cibles pertinents pour en 117 évaluer l'impact. Pour cela, trois panels de bioessais thématiques et fonctionnels ont été choisis : toxicité 118 générale, génotoxicité, perturbation endocrinienne. L'ensemble des bioessais a été appliqué à un large 119 spectre d'eaux rencontrées en milieu urbain afin d'évaluer leur performance et déterminer si certains d'entre 120 eux pourraient s'avérer plus pertinents que d'autres en fonction du contexte. La durée d'exposition des 121 bioesssais varie d'un test à l'autre pour prendre en compte la réponse biologique de l'organisme ciblé. La 122 stratégie adoptée est de type "Whole Effluent Testing" dans laquelle les eaux sont filtrées à 0,22 $\mu \mathrm{m}$ afin 123 d'éliminer les microorganismes présents dans l'échantillon brut. Les tests sont réalisés dans une gamme de $124 \mathrm{pH}$ compris entre 5,5 à 8 et celui-ci peut être ajusté le cas échéant. Chaque bioessai a été réalisé en 125 triplicat. Le tableau 1 présente les campagnes d'échantillonnage et le tableau 2 les éléments 126 méthodologiques relatifs aux bioessais.

\section{$127 \quad 2.1$ Sites d'études}

128 Quarante échantillons ont été collectés à l'échelle de l'agglomération parisienne par les équipes techniques 129 de la ville de Paris et du SIAAP (Figure 1). Ces échantillons concernent un hôpital parisien (HOP, $n=1$ ), les 130 exutoires de deux bassins versants (BV) situés dans les $16^{e}(B V 1, n=3)$ et ${ }^{\circ}(B V 2, n=3)$ arrondissements 131 de Paris, les principaux émissaires $(E M, n=11)$ du SIAAP pour les eaux résiduaires urbaines (tous situés à

\footnotetext{
${ }^{1}$ Note technique du 12/08/16 relative à la recherche de micropolluants dans les eaux brutes et dans les eaux usées traitées de stations de traitement des eaux usées et à leur réduction (BO du MEEM n 2016/15 du 25 août 2016)
} 
l'Ouest de Paris), trois déversoirs d'orage ( $D O, n=5)$ de La Briche, Clichy et Alma, et, enfin, les eaux usées en entrée des STEU (ENT_STEU, n=11) de Seine Amont (SAM), Marne Aval (MAV) et Seine Centre (SEC)

134 ainsi que les eaux traitées (SOR_STEU, $n=6$ ) de Seine Centre et Seine Aval (SAV). Les deux BV parisiens concernent des zones densément urbanisées du centre de Paris sans grande différence d'activités, même si le BV1 correspond à un quartier plus aisé que le BV2. Les DO assurent l'évacuation des eaux unitaires (mélange d'eaux usées et d'eaux pluviales) excédentaires vers la Seine lors d'événements pluvieux importants. Ceux de Clichy et La Briche représentent plus de $80 \%$ des volumes totaux déversés par les DO du SIAAP.

Les échantillons ont été collectés à l'aide de préleveurs automatiques asservis au débit de mi-2015 à mi2017 (Tableau 1). En dehors des DO, toutes les campagnes ont été réalisées par temps sec. Les quatre événements échantillonnés pour les DO correspondent à des hauteurs de pluie comprises entre 5 et $15 \mathrm{~mm}$. Les prélèvements sont conservés dans des bouteilles en verre ambré à $4^{\circ} \mathrm{C}$ pendant au maximum $24 \mathrm{~h}$ avant la réalisation des bioessais. Les paramètres physico-chimiques classiques ont été mesurés et ont validé la représentativité des échantillons pour chaque type d'eau (Zedek 2016).

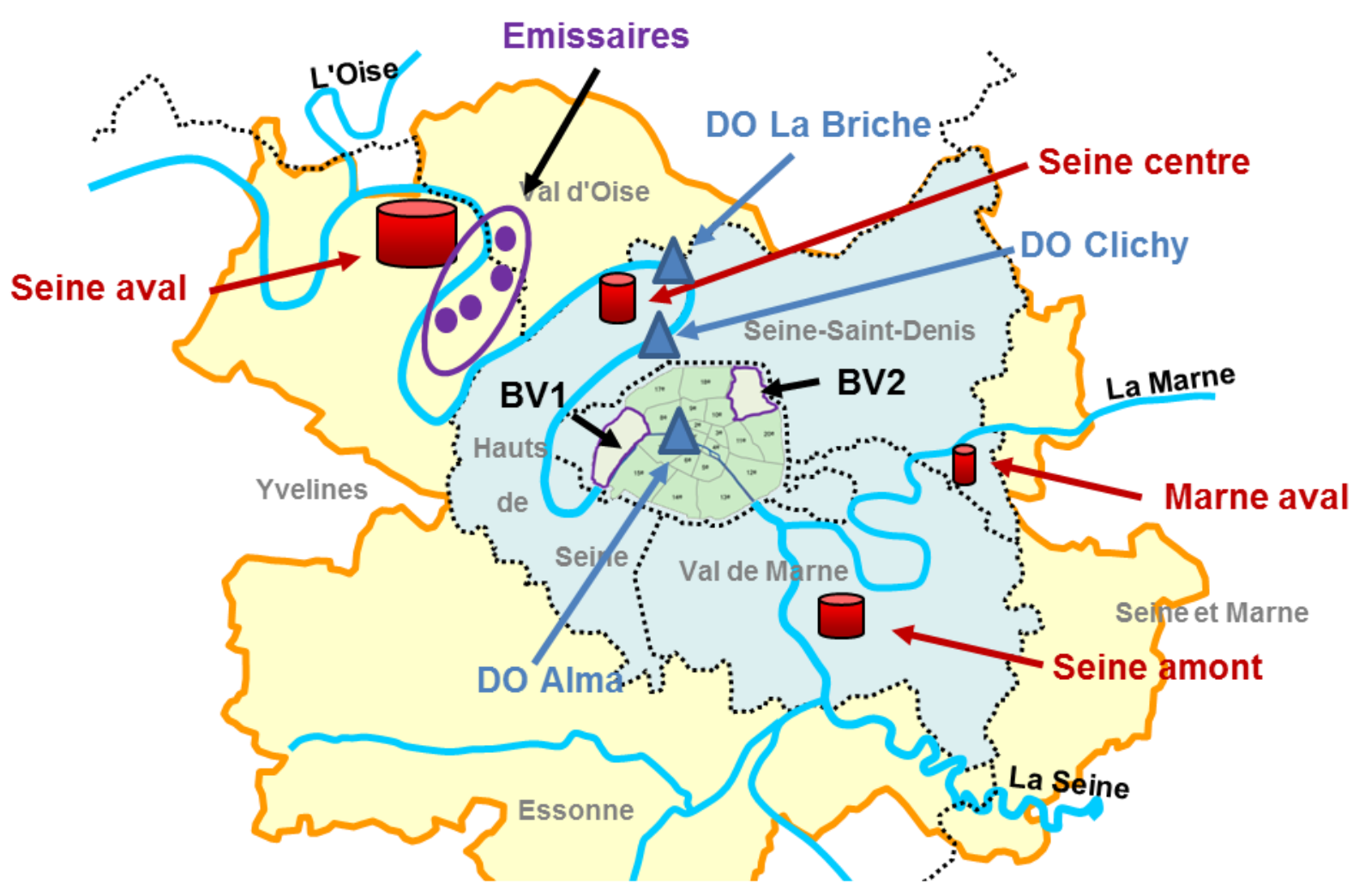

Figure 1. Localisation des sites d'échantillonnage

Tableau 1. Campagnes d'échantillonnage des différents types d'eaux suivies en milieu urbain

\subsection{Panel de toxicité générale}

L'objectif du panel de toxicité générale est d'évaluer la présence d'éléments induisant une toxicité de manière générale, non spécifique et quelle qu'elle soit, sur des organismes cibles variés tels que des bactéries, des algues vertes, des mycètes et des cellules humaines représentant une grande partie de la diversité d'un milieu 
récepteur. Quel que soit l'organisme cible, la toxicité générale est évaluée au travers de la mesure de sa croissance ou de sa viabilité (cytotoxicité). Les différents bioessais de toxicité générale, réalisés sur 3 types d'organismes (bactéries, algues et mycètes), utilisent la même approche d'analyse de leur croissance par turbidimétrie, en mesurant l'absorbance à 600 ou $750 \mathrm{~nm}$ avec un spectrophotomètre. L'absorbance mesurée étant proportionnelle au nombre de cellules présentes dans la suspension test, une relation peut être établie entre le nombre de cellules et la présence de polluants modifiant (accélération ou diminution) cette croissance cellulaire. La mesure de la viabilité cellulaire mise en œuvre sur les cellules humaines est réalisée par ATPmétrie, reflet des cellules fonctionnelles en solution.

163 Les bioessais bactériens sont réalisés sur 2 souches Gram négatives type Escherichia coli sélectionnées sur la base d'antibiogrammes et résistances différentiels: l'une est normale et l'autre sensible. La souche normale a une paroi résistante aux antibiotiques (Ruiz et al., 2005). A contrario, la souche sensible possède une membrane externe défectueuse et perméable, donc plus sensible à certains détergents et antibiotiques ciblant les enzymes de la biosynthèse du peptidoglycane de la paroi bactérienne (Ruiz et al., 2005). Le contraste éventuel entre les réponses de ces souches permet de graduer et nuancer les niveaux de toxicité.

169 Les bioessais sur les algues vertes de la famille des protistes Chlorophytum utilisent deux souches de l'espèce 170 Chlamydomonas reinhardtii (souches sauvage et mutée) qui se différencient par leur sensibilité aux polluants environnementaux en raison d'une mutation affectant la paroi cellulaire. La perturbation (augmentation ou diminution) de la multiplication cellulaire est un marqueur non spécifique répondant à de nombreux polluants (comme des métaux lourds, herbicides, HAP, alkylphénols...). Un effet mesuré sur cet organisme photosynthétique peut mettre en évidence un impact plus général sur l'ensemble de l'écosystème aquatique (Kumar et al., 2014). De plus, la forte conservation des photosystèmes des algues et des plantes supérieures est également intéressante dans l'évaluation de l'impact de substances toxiques chez les végétaux non aquatiques (Juneau et al., 2002 ; Prasad et al., 1998).

Les bioessais sur les mycètes (levures) sont basés sur 2 souches de Saccharomyces cerevisiae, l'une sauvage et l'autre mutante, où les gènes de 7 transporteurs $A B C$ et 2 facteurs de transcription ont été supprimés. Grâce à ces suppressions génétiques multiples, cette souche présente une hypersensibilité à un grand nombre de médicaments comme des antibiotiques, des antiseptiques voire des anticancéreux (Rogers et al., 2001). Un test utilisant la souche de champignon filamenteux ascomycète Mycosphaerella graminicola (Septoria tritici), sensible à de multiples fongicides, est aussi effectué (Ghaffary et al., 2012).

184 La toxicité générale mesurée sur la lignée de cellules humaines (cellules mononucléées du sang périphérique, 185 PBMC) utilise une approche indirecte qui mesure la quantité d'ATP présente dans les cellules métaboliquement actives et, par conséquent, le nombre de cellules viables dans la culture.

\subsection{Panel génotoxicité}

189 Le panel génotoxicité a été conçu pour détecter la présence de molécules capables d'induire soit directement, 190 soit indirectement, des dommages génétiques à des organismes exposés. Un bioessai est réalisé sur des 191 bactéries (souche E.coli RecA de la réponse SOS) et deux sur des cellules eucaryotes (cellules 192 d'adénocarcinome intestinal humain), métabolisant efficacement les bisphénols $A$ et $F$ (Audebert et al., 2011) et des cellules d'adénocarcinome rénal humain, faiblement métabolisantes pour les bisphénols $A, F$ et HAP 
(Audebert et al., 2010). Deux cibles sont donc utilisées pour mettre en évidence les agents génotoxiques/mutagènes, puisqu'un résultat sur un seul type cellulaire n'est pas suffisant pour conclure sur la présence potentielle de molécules génotoxiques dans un échantillon. La génotoxicité a été mesurée par le test H2AX sur les cellules humaines. Les cassures double-brin de l'ADN induisent la phosphorylation de l'histone $\mathrm{H} 2 \mathrm{AX}$ sur la sérine 139. Ce taux de phosphorylation de l'histone $\gamma$-H2AX reflète l'impact génotoxique (Rogakou et al., 1998). La détection de ces cassures de l'ADN double-brin qui sont caractérisées par le rapport H2AX phosphorylée/ADN total, est ainsi 20 à 50 fois plus sensible que le test comètes (Sharma et al., 2012). D'autre part, dans le bioessai bactérien, des bactéries de souche Escherichia coli portant un plasmide dans lequel a été fusionné un promoteur connu pour être activé par des agents génotoxiques (promoteur RecA de la réponse SOS) avec le gène rapporteur luxCDABE de Vibrio fischeri, sont utilisées afin de mettre en évidence l'activation de la signalisation de réparation des dommages à l'ADN grâce à la production de bioluminescence (Rosen et 205 al., 2000).

\subsection{Panel perturbateurs endocriniens}

210 La présence dans les échantillons de molécules capables d'induire des effets perturbateurs endocriniens a été évaluée en utilisant des lignées cellulaires modifiées exprimant un gène rapporteur induisant une luminescence

212 sous contrôle d'un récepteur humain des œstrogènes (ERa), des androgènes ( $A R$ ) ou des hormones 213 thyroïdiennes (TR). Les cellules MELN correspondent à des cellules d'adénocarcinome de sein humain 214 transfectées avec le gène de réponse aux œstrogènes (ERE- $\beta$ Glob-Luc-SVNeo) pour la détection des œstrogènes agonistes (Balaguer et al., 1999 ; Berckmans et al., 2007). Les cellules PALM sont obtenues par transfection de cellules d'adénocarcinome de prostate humain avec le gène du récepteur aux androgènes humain et du gène de la luciférase pour la détection des androgènes agonistes (Escande A., 2009). La lignée HG5LN-TR est obtenue par transfection de cellules avec le plasmide rapporteur Gal4RE5-bGlob-Luc-SVNeo et le récepteur thyroïdien afin de permettre la détection d'agonistes thyroïdiens dans les échantillons (Grimaldi et al., 2015). Ces mesures quantitatives de la perturbation endocrinienne cible ( $E R \alpha, A R$ ou $T R$ ) sont pondérées par des mesures de viabilité cellulaire. Il est important de signaler que les tests antagonistes ne sont interprétables qu'à partir du moment où aucun effet agoniste n'est observable.

\subsection{Exploitation des bioessais}

Chaque bioessai possède ses propres seuils, qui dépendent des caractéristiques de la réponse (prolifération ou toxicité). Ces seuils ont été déterminés en s'appuyant sur la robustesse statistique et les caractéristiques des réponses des modèles (Figure 2). Le passage d'une catégorie à une autre est spécifique à chaque test du panel. Les indicateurs de prolifération sont présents dans les tests impliquant une potentielle multiplication cellulaire et reflètent le fait que les eaux testées peuvent fournir soit des nutriments soit d'autres substances favorisant la prolifération par rapport au contrôle. Les catégories orange et rouge correspondent à une toxicité, générale ou spécifique avérée en fonction du panel. La barre jaune correspond à des réponses certes statistiquement significatives mais toutefois modérées (inférieures à $20 \%$ de réponse au-delà des variations de 
la référence en vert). La couleur noire est utilisée dans les cas où la mortalité cellulaire empêche d'évaluer les réponses des tests de toxicité spécifique (perturbateurs endocriniens ou génotoxicité, par exemple).

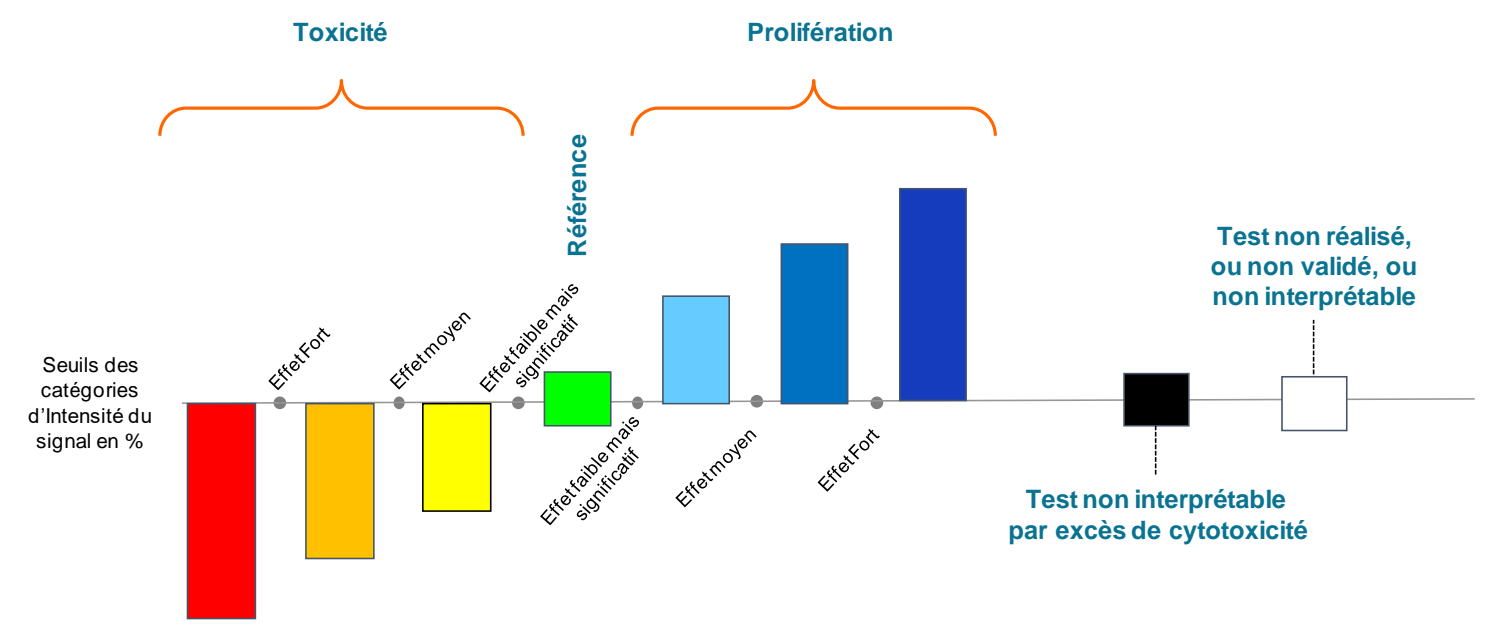

Figure 2. Code couleur utilisé dans l'interprétation des réponses des bioessais des panels utilisés (d'après TameWater)

\section{Résultats et discussion}

240 L'ensemble des résultats des bioessais sont regroupés sous forme de « heatmap ": Figure 3 pour les échantillons issus de HOP, BV et EM, Figure 4 pour les échantillons ENT_STEU et SOR_STEU et, enfin, Figure 5 pour les échantillons appariés de la STEU de Seine centre, c'est-à-dire pour lesquels ENT_STEU et SOR_STEU ont été prélevés le même jour, et les DO.

\subsection{Toxicité générale}

\subsubsection{Bioessais sur souches bactériennes (sauvage et sensible)}

L'ensemble des échantillons (HOP, BV, EM, DO et ENT_STEU) présentent globalement une toxicité très marquée et comparable sur les 2 souches de bactéries, qu'elles soient normales ou perméables. Les échantillons prélevés en sortie de STEU (SOR_STEU) apparaissent peut-être légèrement moins affectés que les échantillons ENT_STEU sur les bactéries normales, mais la toxicité demeure élevée sur les bactéries sensibles. La modification génétique rendant leur paroi défectueuse, elle ne les protège plus aussi bien contre certaines substances dont certains antibiotiques, détergents, colorants...ce qui leur confère une sensibilité non spécifique vis-à-vis de ces substances (Ruiz et al., 2005). Ce test peut donc permettre de conclure sur la présence de telles substances dans ces échantillons, notamment des antibiotiques ainsi que des détergents, fréquemment trouvés dans ces types d'eaux urbaines.

\subsubsection{Bioessais sur algue verte (sauvage et sensible)}

Dans ce test, comme pour le précédent, l'organisme muté utilisé présente une paroi cellulaire déficiente, et est donc plus sensible à la présence de micropolluants (éléments traces métalliques, herbicides, HAP, alkyphénols, etc.), qui peuvent avoir un effet délétère sur l'activité photosynthétique. Les résultats obtenus sont moins homogènes que pour les bioessais sur souches bactériennes; les différents échantillons, selon leurs origines induisent des effets plus variés sur la souche à paroi normale. La souche sensible est 
sur la souche à paroi normale, trois typologies de réponses peuvent être identifiées : un premier groupe constitué de HOP et $\mathrm{BV}$, induisant un effet marqué très fort et négatif, un deuxième groupe avec un effet marqué très fort et positif, et très homogène (DO), un troisième comprenant des réponses variées (positives et négatives selon les dates des prélèvements) pour les échantillons EM et ENT_STEU et SOR_STEU.

266 Ces résultats révèlent : (i) le caractère particulièrement homogène des DO en termes de profil de toxicité, qui induisent systématiquement un impact toxique (pouvant être attribué à des contaminants comme des éléments traces métalliques) ou prolifératif (associé à la présence de nutriments) (Kumar et al., 2014), (ii) la variabilité des profils des EM, ENT_STEU et SOR_STEU, (iii) sur les échantillons prélevés le même jour pour ENT_STEU et SOR_STEU, l'absence d'amélioration de la qualité après la STEU, (iv) un faible effet discriminant du bioessai sur souche sensible (mutante), indiquant un effet toxique systématique sur toutes les eaux, ( $v$ ) une spécificité accrue avec les algues sauvages, si on considère d'une part l'effet positif observé sur la croissance de cet organisme comme résultant de la présence de contaminants, par exemple métalliques, ou d'éléments trophiques, et d'autre part l'effet négatif dû à des substances toxiques pour cette souche représentative à la fois des organismes végétaux aquatiques et non aquatiques. Pour compléter les résultats sur les DO, il serait intéressant d'en cerner l'impact sur le milieu récepteur en appliquant les bioessais en rivière en amont et en aval d'un DO durant un événement.

\subsubsection{Bioessais mycètes (S.tritici, S. cerevisiae)}

Les profils obtenus pour les différents échantillons révèlent beaucoup moins d'effets délétères sur ces organismes eucaryotes que sur les algues sauf de façon marginale (HOP, BV). On retrouve dans une moindre mesure les groupes précédemment évoqués en 3.1.2. Les échantillons provenant des DO se distinguent là-encore des autres pour les deux types de mycètes ( $S$ tritici et $S$ cerevisiae normale, en provoquant leur prolifération), ce qui témoigne de la présence de facteurs contaminants trophiques. La souche de $S$ cerevisiae mutante, de par ses caractéristiques génétiques, est notablement plus sensible que la souche sauvage, comme en témoignent les résultats obtenus avec l'ensemble des prélèvements, qui montrent de façon quasi uniforme un potentiel toxique d'intensité moyenne à forte. Ces résultats sont cohérents avec le fait que $S$ tritici est connu pour être sensible à de nombreux composés. Concernant les seuls échantillons appariés (ENT_STEU et SOR_STEU) de Seine centre, l'abattement apparaît très limité sur $S$ cerevisiae, et sélectif pour $S$ tritici, puisque l'échantillon SOR_STEU induit une prolifération. Un ou plusieurs composés qui inhibaient auparavant la prolifération sont maintenant absents au profit d'autres composés à effet pro-prolifératif. En entrée de STEU, les eaux usées brutes contiennent à la fois des espèces toxiques et des nutriments, l'effet toxique prenant le pas sur l'effet prolifératif. En sortie de STEU, les eaux traitées contiennent moins de contaminants, éliminés par les différents traitements mis en œuvre dans la STEU (Deshayes, 2015 ; Zedek, 2016) favorisant la prolifération des mycètes.

\subsubsection{Bioessai sur cellules humaines (PBMC)}

Les échantillons présentent des effets faibles à moyens sur les PBMC, et ceux-ci peuvent être soit cytotoxiques ou, au contraire, induire leur prolifération. De façon surprenante, les effets globalement toxiques à très toxiques des DO ne sont pas retrouvés sur les PBMC. Concernant les échantillons d'entrée et sortie appariés (ENT_STEU et SOR_STEU), deux types d'effets sont observés. D'une part, ceux-ci ont un effet modérément toxique qui n'est pas abattu dans le rejet pour les prélèvements du 10/10/17, ou induisent 
trouvent significativement réduits dans les rejets (06/02/17). Dans l'ensemble, les effets toxiques semblent très atténués, ce qui montre que ce test n'est pas très sensible mais permet néanmoins de mettre en évidence la présence de facteurs de croissance cellulaire spécifiques des cellules humaines.

\subsection{Génotoxicité}

306 Ce panel est réalisé sur des bactéries et des lignées humaines. L'ensemble des résultats obtenus soulignent que les échantillons montrent une génotoxicité globalement marginale, sauf l'échantillon HOP, au potentiel un peu plus élevé que les autres prélèvements sur la lignée humaine au métabolisme normal. Dans le cadre du projet MICROPOLIS Indicateurs, mené sur le territoire du Syndicat des Bouillides (dans les Alpes Maritimes), Penru et al. (2017) ont mesuré la génotoxicité des eaux usées en différents points du réseau de collecte jusqu'à la sortie de la STEU des Bouillides faisant apparaître eux-aussi un gradient de potentiel génotoxique décroissant de l'amont à l'aval du réseau.

\section{3.3 Perturbation endocrinienne}

314 Dans ce panel, trois types d'agonistes hormonaux sont recherchés : œstrogéniques, androgéniques et 315 thyroïdiens en utilisant des lignées humaines génétiquement modifiées pour la mise en évidence de l'effet 316 supposé. L'ensemble des échantillons testés fait globalement apparaître une activité agoniste très forte 317 d'hormones œstrogéniques. Les activités les moins élevées sont obtenues dans les échantillons 318 SOR_STEU. Concernant les échantillons d'entrée et sortie appariés (ENT_STEU et SOR_STEU), on note 319 un abattement de quasiment la moitié de l'activité (campagne du 10/10/17) contre environ $15 \%$ pour la campagne du 06/02/17. Ces résultats sont cohérents avec ceux de Du Pasquier et al. (2018), qui montrent que l'abattement du potentiel endocrinien est total dans la majorité des cas après l'étape de traitement biologique mais qui mentionnent des abattements ponctuellement médiocres, d'environ $40 \%$, probablement dépendants de la composition de l'effluent considéré. II est cependant difficile de tirer des conclusions définitives sur un nombre réduit de prélèvements appariés, limite mentionnée du fait d'une variabilité du potentiel endocrinien selon les dates de prélèvement (Du Pasquier et al, 2018). De même, Penru et al. (2017) rapportent que l'activité œstrogénique peut présenter des niveaux élevés en entrée de STEU, abattus à plus de $90 \%$ en sortie du traitement tertiaire par ozonation.

328 En ce qui concerne les tests mettant en évidence les effets œstrogéniques antagonistes, du fait de la dominance large de l'effet agoniste dans la quasi-totalité des échantillons quelle que soit leur origine, il n'est pas possible de les quantifier avec précision. Dans ce cas, seule une appréciation qualitative peut être attribuée au test indiquant la présence ou non d'une activité antagoniste. Les deux autres tests, agonistes comme antagonistes androgène/thyroïdien, sont quant à eux globalement non significatifs, sauf cas isolés pour BV, EM, ENT_STEU, et dans des prélèvements relativement contaminés (HOP, DO), ceux-ci demeurant toutefois modestes par rapport aux niveaux observés dans le test agoniste œstrogène. D'une manière générale, les profils d'activités montrent une graduation depuis le réseau d'eaux usées vers la STEU puis vers le milieu naturel. 


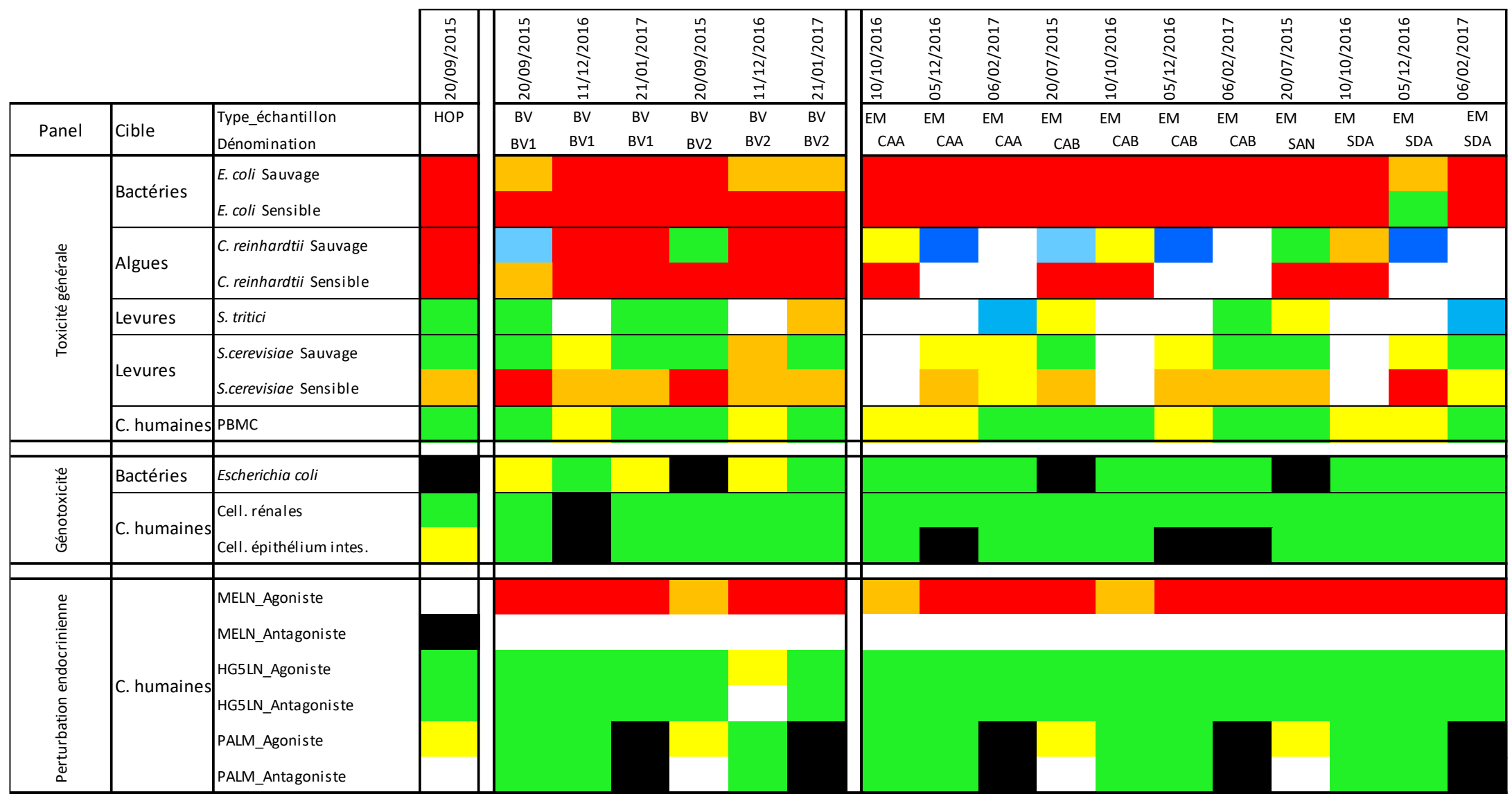

Figure 3. « Heatmap » des potentiels toxiques observés sur les échantillons prélevés en hôpital, à l'exutoire des bassins versants et dans les émissaires 


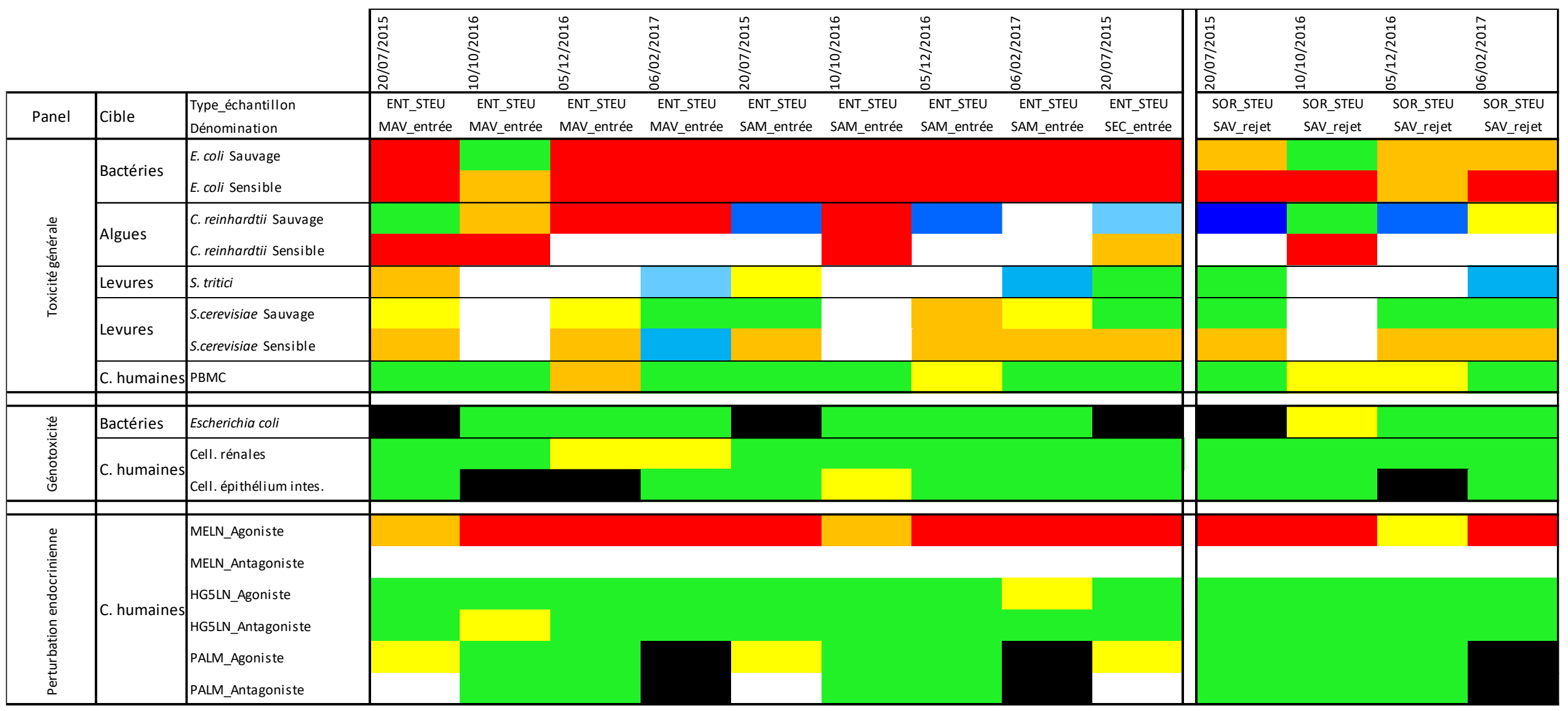

Figure 4. « Heatmap » des potentiels toxiques observés en entrée et sortie des principales STEU de l'agglomération parisienne 


\begin{tabular}{|c|c|c|c|c|c|c|c|c|c|c|c|}
\hline & & & 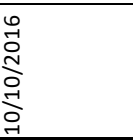 & 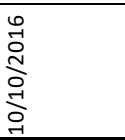 & 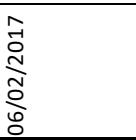 & 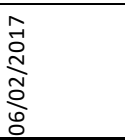 & 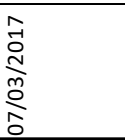 & 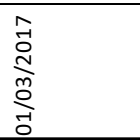 & 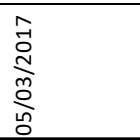 & 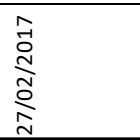 & 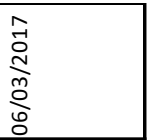 \\
\hline Panel & Cible & \begin{tabular}{|l} 
Type_échantillon \\
Dénomination
\end{tabular} & $\begin{array}{l}\text { ENT_STEU } \\
\text { SEC_entrée }\end{array}$ & $\begin{array}{l}\text { SOR_STEU } \\
\text { SEC_rejet }\end{array}$ & $\begin{array}{l}\text { ENT_STEU } \\
\text { SEC_entrée }\end{array}$ & $\begin{array}{l}\text { SOR_STEU } \\
\text { SEC_rejet }\end{array}$ & $\begin{array}{c}\text { DO } \\
\text { DO_Alma }\end{array}$ & $\begin{array}{c}\text { DO } \\
\text { DO_lichy }\end{array}$ & $\begin{array}{c}\text { DO } \\
\text { DO_lichy }\end{array}$ & $\begin{array}{c}\text { DO } \\
\text { DO_La Briche }\end{array}$ & $\begin{array}{c}\text { DO } \\
\text { Do_La Briche }\end{array}$ \\
\hline \multirow{7}{*}{ 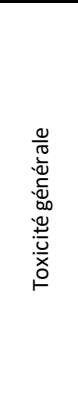 } & Bactéries & $\begin{array}{l}\text { E. coli Sauvage } \\
\text { E. coli Sensible }\end{array}$ & & & & & & & & & \\
\hline & \multirow{2}{*}{ Algues } & c. reinhardtii Sauvage & & & & & & & & & \\
\hline & & c. reinhardtii Sensible & & & & & & & & & \\
\hline & Levures & S. tritici & & & & & & & & & \\
\hline & \multirow{2}{*}{ Levures } & S.cerevisiae Sauvage & & & & & & & & & \\
\hline & & s.cerevisiae Sensible & & & & & & & & & \\
\hline & C. humaines & PBMC & & & & & & & & & \\
\hline \multirow{3}{*}{ 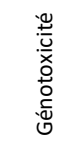 } & Bactéries & Escherichia coli & & & & & & & & & \\
\hline & I bumping & Cell. rénales & & & & & & & & & \\
\hline & & Cell. épithélium intes. & & & & & & & & & \\
\hline \multirow{6}{*}{ 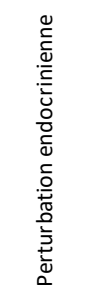 } & \multirow{6}{*}{ C. humaines } & MELN_Agoniste & & & & & & & & & \\
\hline & & MELN_Antagoniste & & & & & & & & & \\
\hline & & HGSLN_Agoniste & & & & & & & & & \\
\hline & & |HG5LN_Antagoniste & & & & & & & & & \\
\hline & & PALM_Agoniste & & & & & & & & & \\
\hline & & |PALM_Antagoniste & & & & & & & & & \\
\hline
\end{tabular}




\section{Conclusion}

351 Notre étude montre que l'approche par panels de bioessais sur des organismes multiples est pertinente pour 352 évaluer l'impact toxique des polluants émergents présents dans différents types d'effluents urbains. Nos 353 résultats permettent d'effectuer un classement des sites étudiés en fonction des réponses biologiques 354 obtenues dans les différents panels, plaçant les effluents des STEU Marne aval et Seine amont largement devant ceux de la STEU Seine centre en termes d'impacts toxiques, tous panels confondus. De même, les déversoirs d'orage apportent un excès de toxicité au milieu récepteur, et les BV présentent des toxicités importantes mais variables, probablement liées aux activités qui y sont menées. Enfin, nos résultats indiquent que l'abattement obtenu pour les paramètres physico-chimiques ne semble pas être retrouvé pour les indicateurs de la toxicité, au moins pour les deux échantillons appariés entrée/sortie étudiés issus de la

360 STEU de Seine centre, ce qui reste à confirmer, ou non, sur un plus grand nombre d'échantillons ou avec 361 d'autres bioessais.

362 Nos résultats permettent également d'atteindre l'objectif d'identifier les bioessais les plus informatifs pour les 363 eaux urbaines : dans le panel Toxicité générale, les tests sur la bactérie sauvage et mutée, l'algue verte 364 sauvage et mutée, le champignon filamenteux et les cellules sanguines humaines apparaissent les plus 365 pertinents pour donner une idée globale du potentiel toxique de l'échantillon au travers d'un choix 366 d'organismes représentatifs et suffisants. Le panel Génotoxicité réagissant de manière faible dans son 367 ensemble, il pourrait se limiter au test bactérien. Cependant, utiliser un seul type d'essai est sujet à générer 368 de faux négatifs (Magdeburg et al, 2014). Enfin, le panel Perturbation endocrinienne peut se suffire de la 369 recherche d'agonistes/antagoniques œstrogéniques, puisque les tests androgènes et thyroïdiens ne 370 répondent que très faiblement ou pas du tout, ce qui témoigne soit d'une sensibilité ou d'une spécificité 371 insuffisante de ces tests à leur présence, masquée par d'autres contaminants, soit d'une absence ou de la 372 rareté de ces composés dans la plupart des prélèvements. Le test œstrogénique agoniste, en englobant des 373 effets agonistes et antagonistes divers, peut refléter plus généralement un potentiel perturbateur 374 endocrinien.

375 II n'y a pas eu la volonté de relier le potentiel toxique observé à la composition chimique de l'échantillon. 376 Zgheib (2009) a montré qu'il était difficile de quantifier l'ensemble des contaminants présents dans un 377 échantillon. La variété et les actions des polluants d'intérêt dépassent largement le cadre des contaminants 378 historiques bien connus tels que les pesticides, HAP, PCB, etc. (Williams et al., 2009). En effet, 379 l'élargissement de la liste des contaminants suivis réalisé par Zgheib (2009) a clairement mis en évidence 380 que les contaminants les plus observés (en fréquence d'observation et/ou concentration) n'étaient pas 381 forcément ceux retenus par la DCE. De plus, il avait été volontairement décidé de ne pas intégrer dans cette 382 liste les produits pharmaceutiques et produits de soins corporels alors qu'il est établi que les eaux 383 résiduaires urbaines véhiculent un large éventail de perturbateurs endocriniens provenant de ces utilisations 384 (Deshayes, 2015 ; Zedek, 2016). Ainsi, il a été démontré que certains de ces composés, tels que les 385 hormones stéroïdiennes naturelles et/ou de synthèse, peuvent contribuer de façon significative à la toxicité 386 des effluents urbains (Cargouët et al., 2004). Un degré de complexité supplémentaire est introduit par 387 l'existence de produits de transformation des médicaments, pour lesquels l'absence d'information, rend la 388 détection et l'évaluation toxicologique impossibles, et dont la toxicité peut excéder celle de la molécule 389 parent (Laurencé et al., 2019). La présence potentielle de l'ensemble de ces composés en cocktail dans les 
prélèvements souligne la nécessité d'en réaliser l'évaluation écotoxicologique en lien avec le potentiel toxique et la composition chimique (screening non ciblé). Ceci permettrait notamment d'obtenir des données sur les effets cocktails qui sont, à ce jour, peu étudiés dans les conditions environnementales. Cette approche plus globale sera appliquée à des prélèvements issus des mêmes sites et constituera l'un des objectifs de la phase 5 du programme OPUR.

\section{Remerciements}

Les travaux présentés ont été réalisés dans le cadre de la $4^{\mathrm{e}}$ phase du programme Observatoire des polluants urbains en Île-de-France (OPUR, financé par l'AESN, le SIAAP, la Ville de Paris, les CD 92, 93 et 94) et du programme Cosmet'eau (Changements de pratiques dans les produits cosmétiques : des lanceurs d'alerte aux impacts sur le milieu aquatique, financé par l'AFB et l'AESN).

\section{Bibliographie}

AIIT-AÏSSA S., MAILLOT-MARÉCHAL E., CREUSOT N., GARDIA-PARĖGE C., BUDZINSKI H. (2017) : Synthèse opérationnelle présentant la détection de composés perturbateurs endocriniens et dioxin-like à l'aide de bioessais in vitro dans les eaux usées, pluviales et naturelles. Projet REGARD (réduction et gestion des micropolluants sur la métropole bordelaise), $8 \mathrm{p}$.

AUDEBERT M., RIU A., JACQUES C., HILLENWECK A., JAMIN E L., ZALKO D., CRAVEDI J. P. (2010) : « Use of the $\mathrm{YH} 2 \mathrm{AX}$ assay for assessing the genotoxicity of polycyclic aromatic hydrocarbons in human cell lines. " Toxicology letters; 199(2): 182-192.

AUDEBERT M., DOLO L., PERDU E., CRAVEDI J. P., ZALKO D. (2011) : « Use of the YH2AX assay for assessing the genotoxicity of bisphenol $\mathrm{A}$ and bisphenol $\mathrm{F}$ in human cell lines. " Archives of toxicology; 85(11): 1463-1473.

BALAGUER P., FRANÇOIS F., COMUNALE F., FENET H., BOUSSIOUX A. M., PONS M., NICOLAS J.-C., CASELLAS C. (1999) : "Reporter cell lines to study the estrogenic effects of xenoestrogens. " Science of the Total Environment; 233(1-3): 47-56.

BERCKMANS P., LEPPENS H., VANGENECHTEN C., WITTERS H. (2007) : " Screening of endocrine disrupting chemicals with MELN cells, an ER-transactivation assay combined with cytotoxicity assessment. » Toxicology in vitro; 21(7): 1262-1267.

418 BOILLOT C. (2008) : Évaluation des risques écotoxicologiques liés aux rejets d'effluents hospitaliers dans 419 les milieux aquatiques. Contribution à l'amélioration de la phase " caractérisation des effets » [thèse]. INSA, 420 Lyon. $298 \mathrm{p}$.

421 BRACK W., ALTENBURGER R., SCHÜÜRMANN G., KRAUSS M., LÓPEZ HERRÁEZ D., VAN GILS J., SLOBODNIK J., MUNTHE J., MANFRED GAWLIK B., VAN WEZEL A., SCHRIKS M., HOLLENDER J., ERIK TOLLEFSEN K., MEKENYAN O., DIMITROV S., BUNKE D., COUSINS I., POSTHUMA L., VAN den BRINK P.J., LÓPEZ DE ALDA M., BARCELÓ D., FAUST M., KORTENKAMP A., SCRIMSHAW M., IGNATOVA S., ENGELEN G., MASSMANN G., LEMKINE G., TEODOROVIC I., WALZ K.H., DULIO V., JONKER M.T.O., JÄGER F., CHIPMAN K., FALCIANI F., LISKA I., ROOKE D., ZHANG X., HOLLERT H., VRANA B., 
H., ESCHER B., DE ARAGÃO UMBUZEIRO G. (2015): "The SOLUTIONS project: Challenges and responses for present and future emerging pollutants in land and water resources management. " Science of the Total Environment; 503-504: 22-31.

BRACK W., AIT AISSA S., BACKHAUS T., DULIO V., ESCHER B. FAUST M., HILSCHEROVA K., HOLLENDER J., HOLLERT H., MULLER C., MUNTHE J., POSTHUMA L., SEILER T-B., SLOBODNIK J., TEODOROVIC I., TINDALL A., DE ARAGAO UMBUZEIRO G., ZHANG X., ALTENBURGER R. (2019) : " Effect-based methods are key. The European Collaborative Project SOLUTIONS recommends integrating effect-based methods for diagnosis and monitoring of water quality. " Environmental Sciences Europe; 31(1): 10.

CARGOUËT M, PERDIZ D, MOUATASSIM-SOUALI A, TAMISIER-KAROLAK S, LEVI Y. (2004). " Assessment of river contamination by estrogenic compounds in Paris area (France). " Science of the Total Environment; 324(1-3): 55-66.

CHARISSOU A.-M., JOURDAIN M.-J., PANDAR P., POULSEN V., DEVILLERS J., FERARD J.-F., BISPO A. (2006) : «Démarche optimale de sélection de batterie de bioessais pour l'évaluation écotoxicologique des milieux complexes. Synthèse bibliographique » Techniques Sciences Méthodes 5: 101-110.

CONNON R.E., GEIST J., WERNER I. (2012): "Effect-Based tools for monitoring and predicting the ecotoxicological effects of chemicals in the aquatic environment » Sensors; 12(9) : 12741-12771.

DU PASQUIER D., LEMKINE G., MEYNEROL K., SAUVIGNET P., BORSATO J., GONCALVES A., ROCHER V. (2015) "Intérêt de la mesure biologique dans le suivi des performances de traitement des polluants «émergents» en eaux résiduaires municipales ». Techniques Sciences Méthodes; 10:, 33-42.

DU PASQUIER D., GUERIN-RECHDAOUI S., AZIMI S., FERAUDET A., LEMKINE G., ROCHER V. (2018) : Évolution de la perturbation endocrinienne des eaux usées lors de leur traitement en STEP - Utilisation des modèles WATCHFROG, in Innover dans les pratiques de monitoring et d'exploitation des stations d'épuration - Enseignements scientifiques et techniques tirés de la phase I du programme MOCOPÉE (2014-2017), 117-127.

DESHAYES S. (2015) : "Identification des sources de phtalates et d'alkylphénols (polluants émergents) en milieu urbain et compréhension des processus d'élimination, [thèse]. Université Paris-Est, Créteil. 252 p.

EUROPEAN COMMISSION (2000) : Directive $n^{\circ}$ 2000/60/CE du 23/10/00 établissant un cadre pour une politique communautaire dans le domaine de l'eau. JO-EU L 327: 1-72.

escande A., SERVANT N., RABENOElinA F., AUZOU G., KLOOSTERbOeR H., CAVAillès V., BALAGUER P., Maudelonde, T. (2009) : « Regulation of activities of steroid hormone receptors by tibolone and its primary metabolites » The Journal of steroid biochemistry and molecular biology; 116(1-2): 8-14.

GHAFFARY S. M. T., FARIS J. D., FRIESEN T. L., VISSER R. G., VAN DER Lee T. A., ROBERT O., KEMA wheat. » Theoretical and Applied Genetics; 124(1): 125-142. GRIMALDI M., BOULAHTOUF A., DELFOSSE V., THOUENNON E., BOURGUET W., BALAGUER P. (2015) : " Reporter cell lines for the characterization of the interactions between human nuclear receptors and endocrine disruptors. » Frontiers in endocrinology; 6: 62. 
JAROŠOVÁ B., BLÁHA L., GIESY J.P., HILSCHEROVÁ K. (2014) : « What level of estrogenic activity determined by in vitro assays in municipal waste waters can be considered as safe? „ Environ.Int. 64: 98109.

JUNEAU P., EL BERDEY A., POPOVIC R. (2002) : " PAM fluorometry in the determination of the sensitivity of Chlorella vulgaris, Selenastrum capricornutum, and Chlamydomonas reinhardtii to copper " Archives of environmental contamination and toxicology; 42(2): 155-164.

KUMAR K. S., DAHMS H. U., LEE J. S., KIM H. C., LEE W. C., SHIN K. H. (2014) : « Algal photosynthetic responses to toxic metals and herbicides assessed by chlorophyll a fluorescence. "Ecotoxicology and environmental safety; 104: 51-71.

LAGARRIGUE C., BRELOT E., NOURY D., SERVETO F., VAN PAASSEN P., WEPIERRE N. (2018) : «Réduction à la source des micropolluants sur le bassin Rhône-Méditerranée : stratégie, enseignements et perspectives. » Techniques Sciences Méthodes 6: 59-70.

LAURENCÉ C., ZEGHBIB N., RIVARD M., LEHRI-BOUFALA S., LACHAISE I., BARAU C., LE CORVOISIER P., MARTENS T., GARRIGUE-ANTAR L., MORIN C. (2019) : " A new human pyridinium metabolite of furosemide, inhibitor of mitochondrial complex I, is a candidate inducer of neurodegeneration. » Biochem Pharmacol.;160:14-23

LEUSCH F. D. L., KHAN S. J., LAINGAM S., PROCHAZKA E., FROSCIO S., TRINH T., CHAPMAN H. F., 483 HUMPAGE A. (2014): " Assessment of the application of bioanalytical tools as surrogate measure of chemical contaminants in recycled water » Water Research; 49: 300-315.

LOOS, R., CARVALHO R., ANTÓNIO D. C., COMERO S., LOCORO G., TAVAZZI S., PARACCHINI B., GHIANI M., LETTIERI T., BLAHA L., JAROSOVA B., VOORSPOELS S., SERVAES K., HAGLUND P., FICK J., LINDBERG R. H., SCHWESIG D., GAWLIK B. M. (2013): « EU-wide monitoring survey on emerging polar organic contaminants in wastewater treatment plant effluents ». Water Research; 47(17): 6475-6487.

MAGDEBURG A., STALTER D., SCHLUSENER M, TERNEST, OEHLMANN J. (2014): « Evaluating the efficiency of advanced wastewater treatment: Target analysis of organic contaminants and (geno-)toxicity assessment tell a different story » Water Research; 50: 35-47.

MOILLERON R., BERGE A., DESHAYES S., ROCHER V., EUDES V., BRESSY A. (2019) : " Importance des émissions d'origine domestique dans les réseaux d'assainissement urbains : cas des alkylphénols, phtalates et parabènes dans l'agglomération parisienne ». Techniques Sciences Méthodes; 5: 75-88.

NELSON J., BISHAY F., VAN RIIDSELAAR A., IKONOMOU M., LAW F. C. (2007). " The use of in vitro bioassays to quantify endocrine disrupting chemicals in municipal wastewater treatment plant effluents ». Science of the Total Environment; 374(1): 80-90.

PENRU Y., GUILLON A., AïT AISSA S., COUTEAU J. (2017) : Caractérisation de la toxicité des eaux usées à Sophia Antipolis. Projet MICROPOLIS Indicateurs : Livrable Nº1, 66 p.

PRASAD M. N. V., DREJ K., SKAWIŃSKA A., STRAŁKA K. (1998) : « Toxicity of cadmium and copper in Chlamydomonas reinhardtii wild-type (WT 2137) and cell wall deficient mutant strain (CW 15). » Bulletin of 
environmental contamination and toxicology; 60(2): 306-311.

503 PRASSE C., STALTER D., SCHULTE-OEHLMANN U., OEHLMANN J., TERNES T. A. (2015) : « Spoilt for 504 choice: a critical review on the chemical and biological assessment of current wastewater treatment 505 technologies. » Water research; 87: 237-270.

506 ROGAKOU E. P., PILCH D. R., ORR A. H., IVANOVA V. S., BONNER W. M. (1998) : « DNA double-stranded breaks induce histone H2AX phosphorylation on serine 139. " Journal of biological chemistry; 273(10): 5858-5868.

ROGERS B., DECOTTIGNIES A., KOLACZKOWSKI M., CARVAJAL E., BALZI E., GOFFEAU A. (2001) : « The pleitropic drug $A B C$ transporters from Saccharomyces cerevisiae. " Journal of molecular microbiology and biotechnology; 3(2): 207-214.

ROSEN R., DAVIDOV Y., LAROSSA R. A., BELKIN S. (2000) : " Microbial sensors of ultraviolet radiation based on recA':: lux fusions. " Applied biochemistry and biotechnology; 89(2-3): 151-160.

RUIZ N., FALCONE B., KAHNE D., SILHAVY T. J. (2005) : "Chemical conditionality: A genetic strategy to probe organelle assembly. »Cell;121(2): 307-317.

SHARMA A., SINGH K., ALMASAN A. (2012): " Histone H2AX phosphorylation: a marker for DNA damage.» In DNA repair protocols (pp. 613-626). Humana Press, Totowa, NJ.

518 TANG J. Y. M., BUSETTI F., CHARROIS J. W. A. ESCHER B. I. (2014) : «Which chemicals drive biological effects in wastewater and recycled water? » Water Research 60: 289-299.

520 WERNERSSON A.-S., CARERE M., MAGGI C., TUSIL P., SOLDAN P., JAMES A., SANCHEZ W., DULIO V., 521 BROEG K., REIFFERSCHEID G., BUCHINGER S., MAAS H., VAN DER GRINTEN E., O'TOOLE S., AUSILI A., MANFRA L., MARZIALI L., POLESELLO S., LACCHETTI I., MANCINI L., LILJA K., LINDEROTH M., LUNDEBERG T., FJÄLLBORG B., PORSBRING T., LARSSON D.J., BENGTSSON-PALME J., FÖRLIN L., KIENLE C., KUNZ P., VERMEIRSSEN E., WERNER I., ROBINSON C.D., LYONS B., KATSIADAKI I., WHALLEY C., den HAAN K., MESSIAEN M., CLAYTON H., LETTIERI T., CARVALHO R.N., GAWLIK B.M., HOLLERT H., DI PAOLO C., BRACK W., KAMMANN U., KASE R. (2015) : « The European technical report on aquatic effect-based monitoring tools under the water framework directive. " Environmental Sciences Europe, 27(7): 11 p. https://doi.org/10.1186/s12302-015-0039-4

WILLIAMS ES, PANKO J, PAUSTENBACH DJ. (2009): « The European Union's REACH regulation: a review of its history and requirements. » Crit Rev Toxicol; 39(7): 553-75.

531 ZEDEK S. (2016): Dynamique de polluants émergents (parabènes, triclosan et triclocarban) dans le continuum eaux grises - milieu récepteur [thèse]. Université Paris-Est, Créteil. $244 \mathrm{p}$.

533 ZGHEIB S. (2009) :. Flux et sources des polluants prioritaires dans les eaux urbaines en lien avec l'usage du 534 territoire [thèse], École nationale des ponts et chaussées, $349 \mathrm{p}$. 
Tableau 1. Campagnes d'échantillonnage des différents types d'eaux suivies en milieu urbain

\begin{tabular}{|c|c|c|c|c|c|}
\hline Échantillon & Site & \multicolumn{4}{|c|}{ Dates de prélèvement } \\
\hline Hôpital (HOP) & & $20 / 09 / 2015$ & & & \\
\hline \multirow{2}{*}{$\begin{array}{l}\text { Bassins } \\
\text { versants (BV) }\end{array}$} & 16e arrondissement (BV1) & \multirow{2}{*}{$20 / 09 / 2015$} & \multirow{2}{*}{$11 / 12 / 2016$} & \multirow{2}{*}{$21 / 01 / 2017$} & \\
\hline & 19e arrondissement (BV2) & & & & \\
\hline \multirow{4}{*}{$\begin{array}{l}\text { Emissaires } \\
\text { (EM) }\end{array}$} & Sèvres Achères Nanterre (SAN) & \multirow{2}{*}{$20 / 07 / 2015$} & & & \\
\hline & Clichy Achères Bezons (CAB) & & \multirow{3}{*}{$10 / 10 / 2016$} & \multirow{3}{*}{$05 / 12 / 2016$} & \multirow{3}{*}{ 06/02/2017 } \\
\hline & Saint-Denis Achères (SDA) & & & & \\
\hline & Clichy Achères Argenteuil (CAA) & & & & \\
\hline \multirow{3}{*}{$\begin{array}{l}\text { Déversoirs } \\
\text { d'orage (DO) }\end{array}$} & La Briche (LB) & $27 / 02 / 2017$ & 06/03/2017 & & \\
\hline & Clichy (CL) & $01 / 03 / 2017$ & $05 / 03 / 2017$ & & \\
\hline & Alma (AL) & & & $07 / 03 / 2017$ & \\
\hline \multirow{3}{*}{$\begin{array}{l}\text { Entrée STEU } \\
\text { (ENT_STEU) }\end{array}$} & Seine amont (SAM) & \multirow{3}{*}{$20 / 07 / 2015$} & \multirow{3}{*}{$10 / 10 / 2016$} & \multirow{3}{*}{$05 / 12 / 2016$} & \multirow{3}{*}{$06 / 02 / 2017$} \\
\hline & Marne Aval (MAV) & & & & \\
\hline & Seine Centre (SEC) & & & & \\
\hline \multirow{2}{*}{$\begin{array}{l}\text { Rejet STEU } \\
\text { (SOR_STEU) }\end{array}$} & Seine aval (SAV) & $20 / 07 / 2015$ & \multirow{2}{*}{$10 / 10 / 2016$} & $05 / 12 / 2017$ & \multirow{2}{*}{$06 / 02 / 2017$} \\
\hline & Seine Centre (SEC) & & & & \\
\hline
\end{tabular}


Tableau 2. Éléments méthodologiques relatifs aux différents bioessais réalisés

543

\begin{tabular}{|c|c|c|c|c|c|}
\hline Panel & Organisme & Témoin positif & Durée & Effet mesuré & Mesure \\
\hline \multirow[t]{8}{*}{ Toxicité générale } & $\begin{array}{l}\text { Bactérie } E \text {. coli } \\
\text { sauvage }\end{array}$ & Ampicilline & $7 \mathrm{~h}$ & Croissance & $\begin{array}{l}\text { Absorbance à } \\
600 \mathrm{~nm}\end{array}$ \\
\hline & $\begin{array}{l}\text { Bactérie } E \text {. coli } \\
\text { sensible }\end{array}$ & Ampicilline & $7 \mathrm{~h}$ & Croissance & $\begin{array}{l}\text { Absorbance à } \\
600 \mathrm{~nm}\end{array}$ \\
\hline & Levure S. tritici & Cadmium & $7 \mathrm{j}$ & Croissance & $\begin{array}{l}\text { Absorbance à } \\
600 \mathrm{~nm}\end{array}$ \\
\hline & $\begin{array}{l}\text { Algue } C . \\
\text { reinhardtii } \\
\text { sauvage }\end{array}$ & Diuron & $48 \mathrm{~h}$ & Croissance & $\begin{array}{l}\text { Absorbance à } \\
600 \mathrm{~nm}\end{array}$ \\
\hline & $\begin{array}{l}\text { Algue } C . \\
\text { reinhardtii } \\
\text { sensible }\end{array}$ & Diuron & $48 \mathrm{~h}$ & Croissance & $\begin{array}{l}\text { Absorbance à } \\
600 \mathrm{~nm}\end{array}$ \\
\hline & $\begin{array}{l}\text { Levure } S . \\
\text { cerevisiae } \\
\text { sauvage }\end{array}$ & Cadmium & $24 \mathrm{~h}$ & Croissance & $\begin{array}{l}\text { Absorbance à } \\
600 \mathrm{~nm}\end{array}$ \\
\hline & $\begin{array}{l}\text { Levure } S \text {. } \\
\text { cerevisiae } \\
\text { sensible }\end{array}$ & Cadmium & $24 \mathrm{~h}$ & Croissance & $\begin{array}{l}\text { Absorbance à } \\
600 \mathrm{~nm}\end{array}$ \\
\hline & $\begin{array}{l}\text { Cellules } \\
\text { humaines } \\
\text { PBMC }\end{array}$ & Cadmium & $24 \mathrm{~h}$ & Viabilité & Luminescence \\
\hline \multirow[t]{3}{*}{ Génotoxicité } & $\begin{array}{l}\text { Cellules LS174T } \\
\text { Cellules } \\
\text { intestinales }\end{array}$ & Benzo[a]pyrène & $72 \mathrm{~h}$ & $\begin{array}{l}\text { Phosphorylation de } \\
\text { I'histone } \mathrm{H} 2 \mathrm{AX}\end{array}$ & Fluorescence \\
\hline & $\begin{array}{l}\text { Cellules ACHn } \\
\text { Cellules rénales }\end{array}$ & Etoposide & $72 \mathrm{~h}$ & $\begin{array}{l}\text { Phosphorylation de } \\
\text { l'histone } \mathrm{H} 2 \mathrm{AX}\end{array}$ & Fluorescence \\
\hline & Bactérie E. Coli & Acide nalidixique & $3 \mathrm{~h}$ & Expression du gène recA & Luminescence \\
\hline \multirow[t]{3}{*}{$\begin{array}{l}\text { Perturbation } \\
\text { endocrinienne }\end{array}$} & Cellules MELN & $17 \beta$-œstradiol & $18 \mathrm{~h}$ & $\begin{array}{l}\text { Activation du récepteur } \\
\text { humain } \alpha \text { aux oestrogènes } \\
\text { (ER } \alpha) \\
\text { (agoniste/antagoniste) }\end{array}$ & Luminescence \\
\hline & Cellules PALM & $\begin{array}{l}\text { R1881 } \\
\text { (méthyltriénolone) }\end{array}$ & $18 \mathrm{~h}$ & $\begin{array}{l}\text { Activation du récepteur } \\
\text { humain aux androgènes } \\
\text { (AR) (agoniste/antagoniste) }\end{array}$ & Luminescence \\
\hline & $\begin{array}{l}\text { Cellules HG5LN- } \\
\text { TR }\end{array}$ & $\begin{array}{l}\text { T3 } \\
\text { (triiodothyronine) }\end{array}$ & $18 \mathrm{~h}$ & $\begin{array}{l}\text { Activation du récepteur } \\
\text { humain aux stéroïdes } \\
\text { thyroïdiens (TR) } \\
\text { (agoniste/antagoniste) }\end{array}$ & Luminescence \\
\hline
\end{tabular}

